\title{
A EDUCAÇÃO INTERPROFISSIONAL, APRENDIZAGEM SIGNIFICATIVA E A PRÁTICA COLABORATIVA NO CENÁRIO DAS POLÍTICAS INDUTORAS DE REORIENTAÇÃO DA FORMAÇÃO EM SAÚDE
}

\author{
INTERPROFESSIONAL EDUCATION, MEANINGFUL LEARNING AND \\ COLLABORATIVE PRACTICE IN THE SCENARIO OF INDUCTIVE POLICIES FOR \\ REORIENTING HEALTH EDUCATION
}
Nara Maria Holanda de Medeiros ${ }^{1}$, Ana Claudia Camargo Gonçalves Germani ${ }^{2}$, Evelyse dos Santos Lemos ${ }^{3}$

Recebido: fevereiro/2021 Aprovado: abril/2021

\begin{abstract}
Resumo: Este artigo discute a Formação em Saúde em uma perspectiva inovadora e provém de uma pesquisa de abordagem qualitativa e quantitativa, que avalia a concepção de discentes egressos do Programa Nacional de Reorientação da Formação Profissional em Saúde (Pró-Saúde) e Programa de Educação pelo Trabalho para Saúde (PET-Saúde) em relação a participação no processo ensino/aprendizagem/avaliação das instituições de ensino superior do Estado de São Paulo, Brasil. Fundamenta-se nas perspectivas da educação interprofissional, aprendizagem significativa, trabalho em equipe e práticas colaborativas. A concepção ampliada de saúde a partir da superação do modelo flexneriano de ensino se faz necessária para qualificação da assistência à saúde. A interdisciplinaridade e a interprofissionalidade são estimuladas a fim de superar esse descompasso e visando a investigação dessa situação, aplicou-se instrumento tipo likert e perguntas abertas à 50 egressos dos Programas. Os resultados evidenciaram o desenvolvimento de competências para interprofissionalidade e atuação com crítica, através do reconhecimento dos princípios, conceitos, pressupostos e de ações conjuntas que estimularam reformas curriculares, contudo, prevalece a desigualdade de acesso aos Programas. Assim, esse artigo reforça a necessidade de interferência das Políticas Indutoras de Reorientação da Formação em Saúde para a qualificação dos processos formativos e assistenciais da saúde.
\end{abstract}

Palavras-chave: Educação Interprofissional. Aprendizagem Significativa. Educação em Saúde.

Abstract: This article discusses Health Education in an innovative perspective and comes from a qualitative and quantitative research, which evaluates the conception of students graduating from the National Program for the Reorientation of Vocational Education in Health Care (Pró-Saúde) and the Education Program for Work in Health Care (PET-Saúde) in relation to participation in the teaching/learning/evaluation process of higher education institutions in the State of São Paulo, Brazil. It is based on the perspectives of interprofessional education, meaningful learning,

http://lattes.cnpq.br/7622095686832163 - Doutorado em Ciências da Saúde pela Universidade Federal de São Paulo (UNIFESP), Brasil. Professora Convidada do Departamento de Medicina Preventiva da Faculdade de Medicina da Universidade de São Paulo e Coordenadora Pedagógica do Programa de Residência Multiprofissional em Atenção Primária à Saúde do UNIFIP/Prefeitura Patos-PB, nm.holanda.medeiros@uol.com.br.

http://lattes.cnpq.br/6041832326296398 - Doutorado em Patologia pela Universidade de São Paulo (USP). Professora do Departamento de Medicina Preventiva da Faculdade de Medicina da Universidade de São Paulo. Av. Dr. Arnaldo, 455, Pinheiros, cep: 01246-900, São Paulo, SP, Brasil. E-mail: accggermani@usp.br.

https: //orcid.org/0000-0003-1024-5290 - Doutorado em Ensino de Ciências pela Universidade de Burgos/Espanha. Docente Permanente do Programa de PG Stricto sensu em Ensino em Biociências e Saúde do Instituto Oswaldo Cruz-Fiocruz, Avenida Brasil, 4365, Manguinhos, CEP: 21.040-360, Rio de Janeiro, Brasil evelyse@ioc.fiocruz.br.

*Agradecimentos especiais, a Dra. Rosana Aparecida Salvador Rossit e Dra. Beatriz Jansen Ferreira, orientadora e coorientadora da pesquisa referida e a Coordenação de Aperfeiçoamento de Pessoal de Nível Superior - Brasil (CAPES) pelo apoio financeiro à pesquisa - Código de Financiamento 001. "This study was financed in part by the Coordenação de Aperfeiçoamento de Pessoal de Nível Superior - Brasil (CAPES) - Finance Code 001". 
teamwork and collaborative practices. The approach with complexity in health is necessary to qualify health care and the uniprofessional ways of acting, in isolation, are insufficient. Interdisciplinarity and interprofessionality are encouraged in order to overcome this gap and aiming to investigate this situation, a likert-type instrument and open questions were applied to 50 graduating students of the Programs. The results showed the development of skills for interprofessionality and critical performance, through the recognition of the principles, concepts, presupposed and joint actions that stimulated curricular reforms, however, inequality of access to the Programs prevails. Thus, this article reinforces the necessary interference of the Inductive Policies for the Reorientation of Health Education for the qualification of health education and assistance processes.

Keywords: Interprofessional Education. Meaningful Learning. Health Education.

\section{Introdução}

O desafio de formar profissionais de graduação em saúde ou de qualificar os profissionais formados permanentemente começa pela compreensão de que ambos não aprenderam de forma compartilhada, significativa, por meio da problematização dos problemas reais, tampouco pelo exercício do próprio protagonismo junto aos processos de aprendizagens.

A educação superior em saúde está marcada pela aprendizagem mecânica, "na qual novas informações são memorizadas de maneira arbitrária, literal e não significativa. Esse tipo de aprendizagem não requer compreensão e não dá conta de situações novas, forma aplicadores e não geradores de conhecimento" (MOREIRA, 2005).

É igualmente preocupante que o ensino superior em saúde, ainda seja marcado por entraves do paradigma formativo Flexneriano de abordagens reducionistas e fragmentadas que limitam as ações integradas em saúde porque não conseguem responder as demandas ampliadas e complexas que envolvem a produção do cuidado integral e interprofissional na saúde.

A ambição do pensamento complexo é dar conta das articulações entre os campos disciplinares que são desmembrados pelo pensamento disjuntivo (um dos principais aspectos do pensamento simplificador); este isola o que separa, e oculta tudo o que religa, interage, interfere. Nesse sentido o pensamento complexo aspira ao conhecimento multidimensional (MORIN, 2011).

A Organização Mundial de Saúde (OMS) salienta, desde 2010, que no atual contexto global, "para se tornar um bom profissional, não basta mais ser profissional, o profissional de saúde também precisa ser interprofissional" (OMS, 2010).

A Educação Interprofissional (EIP) é a estratégia de formação em que "dois ou mais estudantes de profissões diferentes aprendem sobre os outros, com os outros e entre si para melhorar a qualidade do cuidado e a prática colaborativa" (BARR et al., 2016).

A Prática Colaborativa (PC) "ocorre com base na equipe interprofissional, no trabalho em equipe e na integralidade da saúde, envolvendo os pacientes e suas famílias, cuidadores e comunidades em todos os níveis da rede de serviços" (OMS, 2010).

Na produção técnica, Marco para Ação em Educação Interprofissional e Prática Colaborativa, da OMS (2010), a colaboração interprofissional é destacada com potencial para "redução da crise mundial na força de trabalho em saúde, haja vista a carência mundial de 4,3 
milhões de profissionais considerada uma barreira crítica para a conquista dos objetivos de Desenvolvimento do Milênio, pois otimiza os serviços de saúde, fortalece o sistema e incita melhorias de resultados na saúde" (OMS, 2010).

Atualmente, a educação e o trabalho interprofissional estão em consonância com as Diretrizes Curriculares Nacionais (DCN) das profissões da saúde, com as Políticas de Reorientação da Formação em Saúde (BARR, 2015), a Política Nacional de Educação Permanente (PNEP/2018) e com os Planos de Ação da Organização Pan-Americana da Saúde (OPAS) e OMS, desde 2017.

Ressalta-se que a EIP não é uma política nacional de educação, tampouco uma teoria, trata-se de uma estratégia didático-pedagógica, assim sendo, necessita ser praticada com fundamentação teórica consistente. Nas palavras de Barr:

Uma teoria geral da EIP pode um dia tomar forma, entretanto, seus componentes podem ser identificados pela aplicação de princípios da aprendizagem de adultos à aprendizagem interativa, baseada em grupo, que relaciona a aprendizagem colaborativa com a prática colaborativa dentro de uma lógica coerente, fundamentada na compreensão das relações interpessoais, grupais, intergrupais, organizacionais e interorganizacionais e dos processos de profissionalização (Barr, 2005).

Sargeant (2009) aponta para as Teorias Sociais e de aprendizagem, examina a teoria da complexidade e teorias relacionadas à identidade social, entre outras.

Importante destacar a Política Nacional de Educação Permanente em Saúde (PNEPS), portaria 198/GM/2004, como estratégia do Sistema Único de Saúde (SUS) para a formação e o desenvolvimento de trabalhadores para o setor.

O Ministério da Saúde (MS) propõe a Educação Permanente em Saúde (EPS) como estratégia de transformação das práticas de formação, atenção, gestão, formulação de Políticas, participação popular e do controle social no setor saúde e concebe a EPS como a aprendizagem no trabalho, baseada na aprendizagem significativa e na possibilidade de transformar as práticas profissionais. Propõe que os processos de educação se façam a partir da problematização do processo de trabalho, e considera que as necessidades de formação e desenvolvimento dos trabalhadores sejam pautadas pelas necessidades de saúde das pessoas e populações (BRASIL, 2009).

Como pode ser observado, a PNEPS faz referência à base teórica da aprendizagem significativa e metodológica da problematização a partir da definição do próprio conceito de EPS apresentado na sua primeira versão, de 2009, que se mantem na publicação revisada, de 2018. As fundamentações teóricas da PNEPS dialogam com as expressas no pensamento e propostas de Paulo Freire (2005), como pedagogia crítica, prática educativa libertadora e não apenas como método pedagógico, assim como, na perspectiva da aprendizagem significativa expressa o pensamento teórico cognitivista centrado no processo do aprender e no de sua facilitação em contextos educativos (Ausubel, 2003).

Problematizar, na perspectiva da PNEPS, traz o entendimento de repensar sobre as situações e/ou problemas do trabalho, questionando os fenômenos, conhecimentos científicos 
e ideológicos, captando e negociando novos significados, apreendendo e propondo propostas de soluções para serem aplicadas a realidade do trabalho.

Em relação à maneira de aprender significativamente, segundo a teoria de aprendizagem significativa (TAS) de David Ausubel, visão clássica do cognitivismo: "a aprendizagem significativa se caracteriza pela interação - substantiva e não arbitrária - entre o novo conhecimento e o conhecimento prévio, a variável que mais influencia a aprendizagem, seguida da pré-disposição para aprender" (MOREIRA, 2005).

“Na aprendizagem significativa o aprendiz não é um receptor passivo. Longe disso. Ele deve fazer uso dos significados que já internalizou, de maneira substantiva e não arbitrária, para poder captar os significados" (MOREIRA, 2005). Aspecto que dialoga com a prática educativa libertadora de Freire.

Teóricos cognitivistas contemporâneos contribuem e enriquecem a teoria de aprendizagem significativa, entre eles, estão Novak com sua visão humanista, pressupõe aprendizagem como produto da interação de pensamentos, sentimentos e ações e Moreira (2005) com sua visão crítica:

É através da aprendizagem significativa crítica (ASC) que o aluno poderá fazer parte de sua cultura e, ao mesmo tempo, não ser subjulgado por ela, por seus ritos, mitos e ideologias. $E$. através dessa aprendizagem que ele poderá lidar construtivamente com a mudança sem deixar-se dominar por ela, manejar a informação sem sentir-se impotente frente a sua grande disponibilidade e velocidade de fluxo, usufruir e desenvolver a tecnologia sem tornarse um tecnófilo. Por meio dela, poderá trabalhar com a incerteza, a relatividade, a nãocausalidade, a probabilidade, a não-dicotomização das diferenças, com a ideia de que o conhecimento é construção (ou invenção) nossa, que apenas representamos o mundo e nunca o captamos diretamente (MOREIRA, 2005).

A facilitação da ASC ocorre a partir da execução de alguns princípios sistematizados, entre eles: "da interação social e do questionamento; da não centralidade do livro de texto; do aprendiz como perceptor/representador; do conhecimento como linguagem; da consciência semântica; da aprendizagem pelo erro; da desaprendizagem; da incerteza do conhecimento e da não utilização do quadro-de-giz" (MOREIRA, 2005).

Sob o prisma do paradigma de aprendizagem pode-se observar a correlação entre os pressupostos para ASC e a proposta de aprendizagem da PNEPS:

A questão não se limita a incorporar conhecimento, mas saber qual conhecimento é necessário incorporar, o que aprender, o que desaprender e como fazer para que os outros (o grupo como um todo) o façam. O problema vai muito além dos conhecimentos específicos que se ensinam em cursos ou oficinas, e aponta para a mudança nas próprias organizações e seus respectivos contextos (BRASIL, 2009).

A literatura apresenta a forte intersecção do pensamento de Freire (pedagogia crítica) e da teoria de aprendizagem significativa crítica de Moreira apontando que toda ação educativa implica negociação de significados que conduzam aos protagonismos dos sujeitos. 
Visa romper com o paradigma tradicional de ensino, transmissivo, mecânico, acumulativo que alimenta postura passiva dos sujeitos para desenvolver, a partir do paradigma da aprendizagem significativa, uma postura política, reflexiva e metodológica que desperte a curiosidade e o interesse a fim de produzir e sustentar relações em equipe satisfatórias, abordagens interdisciplinares complexas no espaço de trabalho.

Considerando que a EPS, aposta na aprendizagem significativa e na problematização pelo potencial em predispor à aprendizagem de processos complexos como implica o SUS, em mudanças de paradigmas, que Medeiros e Machin $(2011)$ e Medeiros $(2015,2019)$ vem evidenciando em estudos fenomenológicos e outros a efetividade da fundamentação teórica e da aplicação da TAS, visão clássica à crítica e da pedagogia problematizadora de Freire para apoiar a consolidação efetiva da educação e do trabalho interprofissional em saúde, em que se constatou a estimulação permanente da negociação de significados, sentimentos, facilitando a interação com diferentes olhares e a construção de criticidade pela imersão em dinâmicas indagativas que se atribui igual valor aos eixos técnico-científico e ético-humano da formação.

Essa perspectiva também foi enfatizada em âmbito internacional: "As teorias cognitivas, estão estabelecendo quadros úteis na aprendizagem interprofissional em equipe” (IPEC, 2011).

Ressalta-se, ainda, que a PNEPS:

Enfatiza que as demandas para a formação e desenvolvimento dos trabalhadores no SUS não sejam definidas somente a partir de uma lista de necessidades individuais de atualização e da capacidade de oferta e expertise de uma instituição de ensino, mas considerem, prioritariamente, os problemas cotidianos referentes à atenção à saúde e à organização do trabalho (BRASIL, 2009).

A EP e EIP se destacam no cenário brasileiro ao receber o reconhecimento de que "o SUS é interprofissional" (PEDUZZI, 2016), instituído na Constituição Federal de 1988, com os princípios da universalidade, equidade e integralidade, que desafia profissionais e estudantes face às mudanças nos eixos político-administrativo e, sobretudo, paradigmático.

O diferencial do SUS está na proposta de trabalho e aprendizagem a partir do conceito ampliado de saúde, "em que o direito à saúde se confunde com o próprio direito à vida, não mais apenas como ausência de doença" (MEDEIROS, 2019). Nesse sentido, iniciativas de mudança no ensino superior em saúde foram adotadas visando à reorientação da formação.

No ano de 2005, o Governo Federal por meio da parceria entre a Secretaria de Gestão do Trabalho e da Educação na Saúde, MS, Ministério da Educação (MEC) e apoio da OPAS cria o Programa Nacional de Reorientação da Formação Profissional em Saúde (Pró-Saúde), portaria interministerial no 2.102 com a proposta de substituição do modelo tradicional de ensino e assistência, fomenta a integração ensino-serviço, a inovação dos métodos de ensino propondo utilização das metodologias ativas e do trabalho em equipe integrado.

O Pró-Saúde visa catalisar mudanças curriculares, segundo três eixos de transformação: orientação teórica, cenários de práticas e orientação pedagógica e com a mesma finalidade, foi criado o Programa de Educação pelo Trabalho em Saúde (PET-Saúde), em 2008 (MS/MEC), portaria Interministerial no 1802, a fim de fomentar grupos de aprendizagem tutorial na 
Estratégia Saúde da Família, proporcionando a integração de estudantes, docentes, profissionais dos serviços e a população.

Nesse contexto desafiador, tornou-se pertinente analisar a capacidade dos Programas Pró-Saúde e PET-Saúde em se tornarem veículos potenciais para o preparo para o trabalho em equipe interprofissional visando efetivar as PC, de atuação complexa com base na integralidade.

Nessa perspectiva, a pesquisa apresentada analisa a estratégia da EIP, a fundamentação teórica e as práticas desenvolvidas, segundo à concepção dos discentes egressos dos Programas.

\section{Alguns aspectos da Teoria de Aprendizagem Significativa: da visão clássica à crítica.}

Antes da década de 1960, "falávamos de aprendizagem e de não aprendizagem, enquanto atualmente o produto do processo de ensino é analisado como um ponto continuum entre a aprendizagem significativa e a mecânica (LEMOS, 2006).

Aprendizagem significativa é

aprendizagem com atribuição de significados e compreensão [...] com incorporação nãoarbitrária e não-literal de novos conhecimentos à estrutura cognitiva por meio de um processo interativo e progressivo, sob as condições: existência de conhecimentos prévios adequados e predisposição para aprender (MOREIRA, 2008).

A este conhecimento, especificamente relevante à nova aprendizagem, "David Ausubel chamava de subsunçor ou ideia-âncora. Em termos simples, subsunçor é o nome que se dá a um conhecimento específico existente na estrutura de conhecimento de um indivíduo, que permite dar significado a um novo conhecimento" (MOREIRA, 2011).

A maneira mais típica de aprender significativamente é por meio da "aprendizagem significativa subordinada na qual o novo conhecimento adquire significado na ancoragem interativa de algum conhecimento prévio especificamente relevante" (MOREIRA, 2011).

A estrutura cognitiva, considerada como uma estrutura de subsunçores interrelacionadas e hierarquicamente organizados é uma estrutura dinâmica caracterizada por dois processos principais: a diferenciação progressiva e a reconciliação integradora". (MOREIRA, 2011).

A diferenciação progressiva é o processo de atribuição de novos significados a um dado subsunçor resultante da sucessiva utilização desse subsunçor para dar significado a novos conhecimentos. A reconciliação integradora, ou integrativa, consiste em eliminar diferenças aparentes, resolver inconsistências, integrar significados, fazer superordenações, simultâneo a diferenciação progressiva (MOREIRA, 2011).

Joseph Novak, colaborador de Ausubel (1980), "dá a aprendizagem significativa uma conotação humanista, produto da integração construtiva entre pensamento, sentimentos e ações" (MOREIRA, 2011). 
A aprendizagem significativa crítica de Moreira (2005) sustenta que a aprendizagem significativa também seja crítica e pode ser facilitada a partir da execução de alguns pressupostos:

(1) Aprender/ensinar perguntas ao invés de respostas; (2) aprender a partir de distintos materiais educativos; (3) aprender que somos perceptores e representadores do mundo; (4) aprender que a linguagem está totalmente implicada em qualquer e em todas as alternativas humanas de perceber a realidade; (5) aprender que o significado está nas pessoas, não nas palavras; (6) aprender que o homem aprende corrigindo seus erros; (7) aprender a desaprender, a não usar conceitos e estratégias irrelevantes para a sobrevivência; (8) aprender que as perguntas são instrumentos de percepção e que definições e metáforas são instrumentos para pensar; (9) aprender a partir de distintas estratégias de ensino (MOREIRA, 2005).

Essa perspectiva busca preparar o estudante para as mudanças, por meio da transformação do foco do ensino e do foco da aprendizagem que necessita se dar no contexto significativo e ser facilitado no processo de ensino.

\section{Metodologia}

Trata-se de uma pesquisa qualitativa e quantitativa, de caráter exploratório descritivo de avaliação da concepção dos ex-discentes do Pró-Saúde e PET-Saúde que avaliou como eles conceberam sua participação no processo ensino/aprendizagem/avaliação na perspectiva da EIP, ASC, PC e do preparo para o TEI. Essa pesquisa é parte integrante de uma pesquisa mais abrangente de tese de doutorado (2019) da primeira autora do artigo, um Estudo de Caso Avaliativo, constituído de cinco pesquisas para avaliação dos Programas referidos.

A pesquisa está em consonância com os preceitos éticos, firmados na Resolução no 466/2012 e foi aprovado pelo Conselho de Ética.

O contexto da investigação foi as instituições de ensino superior (IES): a Universidade de São Paulo (USP), Universidade Federal de São Paulo (UNIFESP), Pontifícia Universidade Católica de São Paulo (PUC-SP), Faculdade de Ciências Médicas da Santa Casa de São Paulo (SANTA CASASP) e o Centro Universitário Adventista de São Paulo (UNASP). Os ex-discentes referem-se aos alunos egressos da graduação.

Com relação ao número total de ex-discentes que tiveram acesso à participação nos Programas no período investigado pela pesquisa, averiguou-se o total 397 ex-discentes considerando as IES envolvidas. Não foi possível identificar o número total dos ex-discentes que não tiveram acesso aos Programas por IES. Assim, dos 397 ex-discentes participantes dos Programas, 148 eram da USP, 81 da UNIFESP, 38 do UNASP, 30 da PUC-SP e 100 da SANTA CASASP e destes, 50 participaram da pesquisa por meio de consentimento formal que contemplaram o número de indivíduos necessários para garantia de confiabilidade da análise estatística. "As escalas de percepção têm uma dispersão mais adequada em populações de 50 pessoas ou mais, parâmetro padronizado para os estudos psicométricos" (JASEN, 2017). 
A pesquisa de campo consistiu da aplicação do instrumento tipo likert de Avaliação da Interprofissionalidade de Medeiros e Rossit (2018), no grupo dos ex-discentes. Também foi disponibilizado um "espaço aberto" para registro espontâneo de comentários adicionais.

O conteúdo do instrumento likert foi fundamentado, principalmente, na TAS/ASC, a partir das evidências dos estudos de Medeiros e Machin (2011) sobre aplicação das mesmas para efetivação da EIP no contexto do cenário nacional, como apontado pela literatura internacional como uma das prioridades para as novas pesquisas nessa área: "considerar as implicações das estruturas dos serviços nacionais". (BARR, 2005).

Foram classificados em quatro dimensões, o conteúdo da Escala Atitudinal de Avaliação da Interprofissionalidade: (1) conceitual e determinante da EIP; (2) conceitual e determinante da PC; (3) conceitual e determinante do Trabalho em Equipe e do (4) Desenvolvimento de Competência, que agregaram 30 asserções randomizadas.

Também fez parte da coleta de dados a aplicação de um questionário para caracterização do perfil dos participantes que apresentou amostra constituída de 5 profissionais da educação física (10\%), 5 enfermeiros (10\%), 2 farmacêuticos (4\%), 6 fisioterapeutas (12\%), 2 fonoaudiólogos (4\%) 1 médico (2\%), 3 nutricionistas (6\%), 12 psicólogos (24\%), 4 profissionais do serviço social (8\%), 3 terapeutas ocupacionais (6\%) e 7 outros (14\%).

Dos participantes, 40 eram do sexo feminino (80\%) e 10 do sexo masculino (20\%). Quanto a faixa etária, 15 ex-discentes estavam na faixa etária entre 20 a 24 anos (30\%), 27 entre 25 a 29 anos (54\%), 7 entre 30 a 34 anos (14\%), 1 entre 35 a 39 anos (2\%).

Em relação ao ano de ingresso na graduação, 14 ex-discentes realizaram seus cursos entre 2005 a 2010 (28\%), 15 em 2011 (30\%), 9 em 2012 (18\%), 12 entre 2013 a 2017 (24\%). Em relação ao "tempo de atuação" no Pró-Saúde e PET-Saúde, 40 ex-discentes permaneceram de 1 ano a 2 anos (80\%), 4 de 2 anos a 3 anos (8\%) e 6 menos de 1 ano (12\%).

Em relação à Instituição da experiência com Pró-Saúde e PET-Saúde, 21 ex-discentes eram da UNIFESP (42\%), 17 da USP-SP (34\%), 2 da SANTA CASA (4\%), 5 da PUC-SP (10\%) e 5 do UNASP $(10 \%)$, de naturezas jurídicas, pública e privada.

A análise dos dados contou com três etapas, primeiramente, com a submissão das respostas do instrumento tipo likert à uma Análise Estatística, baseada em Ritz (2000) e Schmidt (1975) com teste de confiabilidade de 0,96. Tomou como base as pontuações das asserções e das dimensões que poderiam variar de 1 a 4 . A análise do instrumento likert se baseou em três intervalos de pontuação: de 1 a 1,99 - zona de perigo: a percepção é negativa e as mudanças imediatas devem ser tomadas; de 2 a 2,99 - zona de alerta: a percepção revela os aspectos a serem aprimorados e exige medidas a médio prazo; e, de 3 a 4 -zona de conforto: a percepção é positiva e pode ser potencializada.

Subsequentemente, foi realizado a análise qualitativa interpretativa baseada em André (1988) e Erickson (1989) dos resultados das referidas médias das dimensões e de suas respectivas asserções em relação ao arcabouço teórico e análise dos depoimentos dos 19 exdiscentes que completaram o campo de comentários adicionais de forma dissertativa. Para fins 
de organização, os trechos redigidos pelos ex-discentes estão apresentados no formato alfanumérico, por exemplo, Ex1, Ex2, Ex3, e assim por diante até Ex19.

\section{Apreender o olhar compreendido dos ex-discentes do Pró- Saúde e PET-Saúde}

"Ver não é simplesmente olhar; é, ao mesmo tempo, compreender. É graças a um jogo de atitude que o indivíduo escolhe na realidade o que para ele faz sentido, e isso é tudo que ele retém dela. As atitudes permitem, portanto, fazer uma escolha na realidade" (MENDRAS, 2004). $\mathrm{O}$ instrumento tipo likert permite avaliar um conjunto de dimensões de atitudes que visam investigar como os seus componentes influenciam na mudança de comportamento e percepções (FABRIGAR; WEGENER, 2010).

Atitude, entendida como uma tendência psicológica avaliativa, adquirida, formada por crenças e conhecimentos, influenciada pelas emoções favoráveis ou desfavoráveis no que tange à reação e respostas relacionadas às pessoas, grupos sociais, objetos, comportamentos, percepções ou outros conceitos (FABRIGAR; WEGENER, 2010).

As respostas dos ex-discentes à escala atitudinal de likert quantificam indicadores de percepção, em relação ao nível de concordância e discordância dos respondentes.

O perfil geral atitudinal do desempenho dos ex-discentes pela interpretação dos resultados das referidas médias das dimensões e de suas respectivas assertivas denotaram compreensão e aplicabilidade acerca dos conceitos e do desenvolvimento das práticas interprofissionais, cujos resultados das médias das dimensões foram: D1, conceitual e determinante da EIP- 3,40; D2, conceitual e determinante da PC- 3,33; D3, conceitual e determinante do trabalho em equipe- 3,23; D4, do desenvolvimento de competência- 3,15. Todas encontram-se no intervalo de pontuação 3,00 a 4,00, demonstrando inclinação à concordância, situada na "zona de conforto", reveladora de concepções positivas a serem potencializadas.

Na perspectiva das IES, a partir da concepção dos ex-discentes, elas tornaram possível encontrar espaços para incorporação de módulos compartilhados, estimularam transformações curriculares, incentivaram inovações metodológicas e contemplaram o arcabouço teórico de compreensão e implementação da EIP.

Esses aspectos também foram observados nos seguintes depoimentos:

Acredito que em geral o programa me ajudou [...] no conhecimento de outras áreas pelos projetos desenvolvidos. Em todos os momentos discutíamos o que poderia ser feito, pesquisávamos e também conversávamos sobre a visão da outra profissão (Ex2).

Percebo a potência e o privilégio que tive quando participei do PET-Pró-Saúde [...] aprendi, principalmente sobre trabalhar com pessoas dentro de uma comunidade, atuar no Sistema Único de Saúde e a lidar com as relações de equipe, em especial, na perspectiva interprofissional. O PET me abriu portas únicas. Hoje carrego na minha atuação a rica bagagem de ter vivenciado um processo participativo, prático e reflexivo no PET (Ex9). 
Para Barr,

Os movimentos em EIP têm os mesmos motivos: melhorar a prática, o cuidado do paciente; aprimorar competências profissionais e sobressair-se; adquirir força coletiva e fixar um lugar no ensino superior. Ao aprenderem em conjunto, as profissões trabalharão mais eficazmente em conjunto e, assim, melhorarão a qualidade dos cuidados prestados (BARR, 2005).

A reflexão sobre "o fazer" como um processo cognitivo de qualificação do conhecimento e de competência é um dos aspectos defendidos no trabalho em equipe e reconhecido pelos exdiscentes dos programas: "A experiência não tem preço" (Ex12).

O processo de refletir sobre as práticas atrelado à um processo cognitivo de qualificação do conhecimento revelou o reconhecimento por parte dos ex-discentes da maneira de aprender significativamente pela necessidade da interação seletiva entre o novo material de aprendizagem e as ideias preexistentes na estrutura cognitiva sendo eles "assimilados, diferenciados, elaborados e estabilizados, constituindo uma experiência consciente, articulada e precisamente diferenciada" (MOREIRA; MASINI, 2001).

Sob o prisma da avaliação, foi evidenciado, segundo à concepção dos ex-discentes, que as avaliações nos Programas estiveram implicadas com a visão ampliada do processo saúdedoença, com o desenvolvimento de competências e sob a utilização de diferentes métodos avaliativos que se complementaram (do tipo formativo, recursivo e somativo), de acordo com as situações e/ou problemas conduzidos. Faço eco com o depoimento da egressa:

O PET-Saúde me proporcionou vivenciar situações profissionais, ainda, durante a vida acadêmica, que hoje me fazem atuar de forma mais assertiva e com maior responsabilidade social. Sou muito grata a todos os envolvidos, pois hoje sigo muitos dos exemplos de atuação dos meus professores e preceptores daquela época (Ex5).

Corroboram com a intersecção da EIP e da AS, em que a avaliação da aprendizagem significativa:

Deve avaliar a compreensão, a captação de significados, capacidade de transferência do conhecimento às situações não conhecidas; deve ser predominantemente formativa $e$ recursiva. É importante a recursividade, ou seja, permitir que o aprendiz refaça. É importante que ele externalize os significados que está captando" (MOREIRA, 2011).

“A EIP pressupõe que a avaliação seja rigorosa, a fim de analisar o impacto e identificar evidências, no contexto prático, quanto a aquisição de competências interprofissionais por parte dos aprendizes" (MAHLER et al., 2014).

A capacidade dos ex-discentes "para demonstrar um conjunto integrado de competências e transferir sua aplicação em diferentes contextos e em cada situação, é a medida da sua capacidade de praticar em colaboração" (CIHC, 2010).

A OMS (2010) apontou em seus documentos a estreita intersecção entre a EIP e as PC, apontando um mecanismo de sinergia e interdependência entre essas estratégias culminando com a evidente qualificação do cuidado. 
Um dos benefícios da implementação da EIP e PC é que elas modificam a forma como os profissionais de saúde interagem uns com os outros para prestar assistência. "Ao modificar a forma como os profissionais pensam e interagem, a cultura do ambiente de trabalho e as atitudes da força de trabalho se transformarão, melhorando a experiência de trabalho dos profissionais e beneficiando a comunidade como um todo" (OMS, 2010).

Em relação à dimensão $\mathrm{D} 2$, conceitual e determinante da $\mathrm{PC}$, de pontuação $3.33 \mathrm{e}$ segundo a concepção dos ex-discentes, de inclinação à concordância, revelaram concepções positivas a serem potencializadas e também podem ser conferidas nos depoimentos:

Passei a ver o funcionamento do SUS de forma interna, me inseri em uma equipe multiprofissional e vi como trabalhar em equipe proporciona um melhor acolhimento do paciente e um planejamento amplo que gera impacto de forma expressiva em sua qualidade de vida. Hoje, mesmo tendo trabalhado em clínicas particulares e estando em curso de pósgraduação (Doutorado em ciências odontológicas) sempre busco trabalhar em equipe e de forma multiprofissional, para oferecer o melhor tratamento e atingir resultados mais amplos, de forma mais rápida e efetiva, sendo possivel devido a consideração de diversos pontos de vista e na execução da melhor abordagem considerada (Ex8).

A participação no PET - RUE: Rede Urgência e Emergência Hipertensão Arterial Sistêmica e Diabetes Mellitus, como discente de serviço social noturno, foi uma grande experiência multiprofissional com um maior contato com os serviços públicos da saúde e os profissionais (principalmente pela abertura dos preceptores). Um fator importante e diferenciado foi a autonomia e confiança tanto dos usuários como dos profissionais na realização das intervenções em saúde (Ex4).

Acredito que o trabalho em equipe possibilitou ideias e criatividade nas diferentes situações do cotidiano da equipe e dos usuários. Trouxe resultados alcançados somente por meio da interdisciplinaridade dos cursos, e essa é a maior potencialidade que enxergo já que o trabalho com uma equipe interdisciplinar é completa e desenvolve um trabalho com o usuário que enxerga além do patológico (Ex7).

“A interdisciplinaridade é considerada uma inter-relação e interação das disciplinas a fim de atingir um objetivo comum [...] e construir novos conhecimentos" (VILELA; MENDES, 2003). Corroborando com Feuerwerker e Sena-Chompré (1999):

Um dos desafios da díade multiprofissionalidade e interdisciplinaridade ao longo desse tempo tem sido constituir consistência à interprofissionalidade: entendimento de que o trabalho em saúde requer uma equipe com a presença de várias profissões no campo, em atuação interdisciplinar e em construção contínua das práticas compartilhadas.

Os ex-discentes conceberam a intersecção da interprofissionalidade ou multiprofissionalidade com a interdisciplinaridade como possibilidade de mitigar a distância dos problemas do mundo real e a organização disciplinar do conhecimento pela interação das disciplinas em seu grau mais avançado, a fim de fazê-las integrarem, refletindo a vivência de estratégias que podem levar à superação da tendência à fragmentação do conhecimento e compreensão de um conceito ampliado de saúde exemplificado no contexto das práticas nos 
Programas para as melhorias no cuidado, assim o que se evidenciou foi o reconhecimento do aumento do potencial resolutivo das equipes.

Sob o prisma cognitivista, constatou-se a aprendizagem significativa do novo conceito ampliado de saúde, não mais como sinônimo de ausência de doença, mas de qualidade de vida e outros conceitos relacionados como de integralidade, equidade, cuidado, universalidade, dignidade, cidadania e outros, como pressupõe o SUS no seu eixo paradigmático, na concepção dos ex-discentes. A aquisição e utilização do novo conceito de saúde pelos ex-discentes reflete a "aprendizagem significativa proposicional", cuja tarefa é aprender significados de ideias expressas em forma de proposição/conceitos. A aprendizagem significativa proposicional é do tipo "combinatória", pois caracteriza a situação em que a nova proposição não é relacionada às proposições subordinadas ou superordenadas específicas, e sim com antecedentes amplos de conteúdo relevante existentes na estrutura cognitiva.

A utilização das proposições e/ou conceitos ocorrem pela aquisição de novos conhecimentos, categorização percentual da experiência, solução de problemas e percepção de novos significados dos conceitos e proposições previamente aprendidos. A aquisição e uso de conceitos se sobrepõem em muitos sentidos. Conceitos adquiridos são utilizados na solução de uma variedade de problemas, dos mais simples aos mais complexos dialogando com a perspectiva da EIP na articulação da aprendizagem significativa e da metodologia problematizadora.

A teoria ausubeliana sustenta que os conceitos resultam de uma "experiência" e são produtos "fenomenológicos". Isso subentende um indivíduo ativo numa situação, num processo de elaboração pessoal (MOREIRA; MASINI, 2001) corroborando com a concepção dos exdiscentes em relação sua pró-atividade nos Programas, ou ainda no reconhecimento da "passagem da situação de objeto à sujeito" como preconiza Medeiros (2015) nos pressupostos para o ensino ativo em serviço.

No que diz respeito à média da dimensão D3 (dimensão conceitual e determinante do trabalho em equipe), de pontuação 3.23 , a concepção dos ex-discentes apresentou inclinações à concordância e discordância em relação aos aspectos abordados nas asserções. O conceito de trabalho em equipe que almejamos na formação e trabalho em saúde, refere-se:

Ao processo dinâmico que envolve dois ou mais profissionais de saúde com formação e habilidades complementares, compartilhando objetivos comuns de saúde e exercitando esforços físicos e mentais combinados na avaliação, planejamento ou avaliação do atendimento ao paciente. Isso é realizado por meio de colaboração interdependente, comunicação aberta e tomada de decisão compartilhada. Isso, por sua vez, gera resultados de pacientes, organizações e funcionários com valor agregado (XYRICHIS; REAM, 2008).

A inclinação dos ex-discentes à discordância em resposta à escala atitudinal ocorreu em relação à temática da "integração em equipe" abordadas nas asserções A7 e A11, pontuações 2.98 e 2.90 , de concepções negativas, sugestivas de medidas a médio prazo, todavia esse resultado foi vinculado apenas ao grupo de ex-discentes respondentes que atuaram por "menos de um ano" nos Programas. Dos 50 ex-discentes respondentes, 6 ex-discentes participaram dos Programas por "menos de um ano" ( $12 \%$ da população do estudo) e se averiguou que esse 
intervalo de tempo, por vezes pode ser insuficiente para conclusão das etapas essenciais para a ocorrência da integração efetiva entre membros de equipes da saúde.

Quatro etapas são consideradas essenciais para a ocorrência da integração efetiva entre membros de equipe:

(1) o alcance do estabelecimento de objetivos comuns pactuados democraticamente; (2) o desenvolvimento das ações de saúde a partir do alcance de atuação com os mesmos métodos educativos/reflexivos com os outros membros da equipe; (3) o alcance da atuação a partir das mesmas concepções construídas por divergência e convergência dos seus membros; e (4) pelo estabelecimento de forte sentimento de pertencimento, assim o processo de integração em equipe é analisado, sobretudo, pelo reconhecimento da condição de se tornar "extensão da equipe" (MEDEIROS; MACHIN, 2011).

Trata-se muito mais de criar e consagrar sinergia, do que da situação em que cada indivíduo passa usar suas próprias habilidades para alcançar objetivos comuns (OMS, 2010).

Em contraposição, à concepção dos ex-discentes que participaram por "mais de um ano" nos Programas ( $88 \%$ da população do estudo) evidenciou o reconhecimento e defesa do "modus operandi" da EIP, PC e do trabalho em equipe integrado durante a experiência.

Corroborando com os depoimentos:

"Aprendi principalmente sobre trabalhar com pessoas [...] e a lidar com as relações de equipe, em especial, na perspectiva interprofissional" (Ex9).

"O PET foi a experiência que permitiu que eu entendesse [...] que as equipes de saúde bem treinadas e motivadas são fundamentais para as ações de prevenção e cuidados a saúde da população" (Ex12).

Ressalta-se que o trabalho em equipe é central na EIP, face à relação dinâmica com as necessidades da prática e as melhorias da prática (IPEC, 2011).

A efetividade do preparo para o trabalho em equipe vem sendo frequentemente relacionada, na perspectiva nacional e internacional, com o desenvolvimento das competências. Barr faz distinção entre competências "comuns", aquelas comuns entre todas as profissões, "complementares", as que distinguem uma profissão de outra e as "colaborativas", aquelas necessárias para trabalhar efetivamente com as outras (BARR, 2005).

Nessa perspectiva, a concepção dos ex-discentes evidenciou atuação nos eixos: técnicocientífico e ético-humano, no que tange questões objetivas e subjetivas das relações de ensinoaprendizagem e trabalho. Eles perceberam o desenvolvimento das faculdades cognitivas e emocionais a fim de favorecer os processos de aprendizagem, assim lidar com seus próprios sentimentos e sofrimentos e com os dos outros ocuparam posições no ensino ativo das práticas.

Competência tem sido interpretada no ensino superior em ciências da saúde "como a vinculação de conhecimentos, habilidades, atitudes e valores a fim de proporcionar uma formação integral (CONEJERO et al., 2013). 
O desenvolvimento de competências para a formação ética-humanizada é um dos grandes desafios para consolidação do eixo paradigmático do SUS que implica em mudanças na formação e na Educação Permanente das profissões da saúde, haja vista, que muitos profissionais de saúde, inclusive docentes, não tiveram acesso a essa discussão no contexto das práticas, por meio da problematização de situações-problemas no contexto real de atuação.

Medeiros (2019) reforça a necessidade de que a reforma do pensamento ocorra para além do refinamento intelectual, que passe, sobretudo, pela consideração dos aspectos subjetivos, a partir do desenvolvimento de concepções e competências mais apuradas dos sentidos, haja vista ser este um dos principais empecilhos para formação em saúde e para o preparo para o trabalho em equipe: a supervalorização do desenvolvimento de competências para condução de questões de cunho técnico-científico e ínfima importância dada as questões de valores, ética e humanidades.

Os ex-discentes conceberam que a experiência problematizadora e significativa também favoreceu a compreensão dos aspectos subjetivos e desencadeou o exercício de controlar as próprias emoções de forma a favorecer a aprendizagem e o próprio crescimento pessoal, além de possibilitar a maior compreensão do sofrimento do outro por meio da qualificação dos processos de empatia, liderança, mediação de conflito, comunicação e escuta.

"São coisas que vamos levar para o resto da vida como experiência muito boa e grande crescimento profissional, e abrir os olhos para outras pessoas e outros sofrimentos" (Ex1).

Os ex-discentes com "mais de um ano" de atuação nos Programas (88\% da população do estudo) reconheceram a competência dos docentes de prepará-los para lidar com o sofrimento do outro, enquanto os ex-discentes com "menos de um ano" no Programa ( $12 \%$ da população do estudo) não identificaram essa competência e relacionaram essa fragilidade à falta de EP para os preceptores dos Programas.

Os cenários de prática colocam os estudantes em contato com demonstrações claras dos limites da humanidade, do cuidado com a doença e a morte, ocasionando que eles experienciem suas próprias limitações entre as fragilidades do racional e emocional e como nenhuma atividade é desprovida de emoção, vários sentimentos são emergidos, assim como situações e sensações. São exemplos: dúvidas, decepção, ansiedade, medo, tristeza, raiva e estresse. Este é um momento onde o desconhecido pode interferir na relação de ensino-aprendizagem fazendo necessário o monitoramento do processo pedagógico e avaliativo, sempre almejando aprendizagem significativa do aprendiz.

Junto às mudanças cognitivas ocorrem também alterações dos estados emotivos do aprendiz:

$O$ ato de aprender está implicado com os mais diversos sentimentos e emoções que acompanham o processo de perceber, analisar, comparar, compreender que configuram o ato de aprender. Ao apreendermos algo, um novo conhecimento é construído e assimilado, que desencadeia um melhor processamento mental ou motor e ao mesmo tempo se adquire uma maior confiança na própria capacidade de aprender. (BORDENAVE; PEREIRA, 2008) 
Em relação aos aspectos positivos dos processos subjetivos das relações de trabalho, constatou-se segundo à concepção dos ex-discentes que a vivência nutriu sentimentos de entusiasmo, de conviç̧ões sobre as possibilidades de mudanças. Ofereceu oportunidade para criatividade, melhoria pessoal e das relações interpessoais com base na aprendizagem significativa, haja vista que a aprendizagem mecânica "tem pouca retenção, não requer compreensão e não dá conta de situações novas" (MOREIRA, 2005).

Outros depoimentos conferem o desenvolvimento de competências para além da atuação prática no eixo técnico-científico:

O PET-Saúde é um ótimo programa, que possibilita aos alunos de graduação o aprendizado de habilidades que vão além do currículo e que são imprescindiveis. E ainda proporciona experiências de vida muito inspiradoras para um futuro profissional da saúde" (Ex10).

"Acredito que o trabalho em equipe possibilitou ideias e criatividade nas diferentes situações do cotidiano da equipe e dos usuários" (Ex7).

Corroborando com a visão humanista de Novak, a aprendizagem significativa "ocorre pela integração entre pensamentos, sentimentos e ações e pode ser positiva, negativa ou matizada. Nesse caso, o aprendiz cresce, tem uma boa sensação e se predispõe à novas aprendizagens na área" (MOREIRA, 2011).

A concepção dos ex-discentes em relação à dimensão D4 - do desenvolvimento de competências, de pontuação 3.15 , de concepção positiva, inclinação à concordância, que aprofunda a discussão acerca da temática do desenvolvimento de competências, sobretudo para aquisição e/ou aperfeiçoamento de base política e educativa crítica, revelou que os Programas conseguiram inserir e comprometer os ex-discentes no âmbito da melhor compreensão e comprometimento com o SUS.

“O PET foi a experiência que permitiu que eu entendesse, sem preconceitos, o quão importante o SUS é para nós e que as equipes de saúde bem treinadas e motivadas são fundamentais para as ações de prevenção e cuidados a saúde da população" (Ex12).

Esse é outro aspecto crucial da Formação em Saúde, tendo em vista, que profissionais e estudantes da saúde necessitam adquirir e/ou aperfeiçoar competências para apoiar a reflexão sobre políticas públicas de saúde voltadas para o fortalecimento do SUS.

Todavia, a fragilidade de participação dos profissionais nos canais legítimos de participação popular espelha o desafio da formação em saúde em garantir uma formação mais robusta no tocante à atuação política, metodológica e crítica que permita atuação plena nas diferentes instâncias de políticas públicas de saúde. Para tanto, é necessário incluir os atores sociais da saúde em sucessivos ciclos de trabalho-aprendizagem visando "reconstruir conceitos, refletir os aspectos políticos, econômicos, sociais, ambientais e superar o reducionismo da educação conservadora" (CRUZ et al.,2021).

Na perspectiva da Teoria da Aprendizagem Significativa Crítica (TASC) de Moreira (2005), pode-se dizer que o que se busca é uma formação de consciência sanitária. 
Os ex-discentes reconheceram a dinâmica pedagógica que os inseriu em sucessivos ciclos de questionamentos permanentes em relação as diretrizes e princípios políticos do SUS. No âmbito da estrutura cognitiva, a aprendizagem de conceitos relacionados com a vertente mais política da educação se expressa "na integração de novas informações em um complexo processo pelo qual, situado no tecido dos acontecimentos em suas ações, interações, retroações, e determinações, aquele que aprende adquire conhecimento" (MASINI; MOREIRA, 2008).

"A Aprendizagem Significativa e Crítica é estimulada pela busca de respostas (questionamentos), pelo abandono da narrativa em favor de um ensino centrado no aluno e pela diversidade de materiais e estratégias instrucionais" (MOREIRA, 2011).

Sob o prisma cognitivista:

O conhecimento é produzido em resposta a perguntas; todo novo conhecimento resulta de novas perguntas, muitas vezes novas perguntas sobre velhas perguntas. Quando um aprendiz formula uma pergunta relevante, apropriada e substantiva, ele utiliza seu conhecimento prévio de maneira não-arbitrária e não-literal, e isso é evidência de aprendizagem significativa crítica. (POSTMAN; WEINGARTNER, 1969)

Importante diferenciar que enquanto a educação tradicional produz personalidades passivas e acomodadas, a EIP com base na ASC e no fortalecimento da democracia do cotidiano almejam formar pessoas, com personalidade ativa, flexível, criativa e crítica "que possa enfrentar a incerteza e a ambiguidade sem se perder, e construir novos e viáveis significados para encarar as ameaçadoras mudanças ambientais" (MOREIRA, 2005).

Portanto, no contexto geral das quatro dimensões, segundo à concepção dos ex-discentes do Pró-Saúde e PET-Saúde, concluiu-se o potencial dos Programas para mudar paradigmas e refletiu o quanto as IES tornaram praticáveis a EIP, a aprendizagem significativa e o preparo para o trabalho em equipe interprofissional visando a efetivação das PC. Dessa forma, as reformas curriculares foram estimuladas, situação fundamental para se empreender e sustentar mudanças no pensar e no fazer em saúde.

\section{Considerações finais}

Embora o Pró-Saúde e PET-Saúde caracterizem um terreno fértil para EIP, AS/ASC, para o preparo para o trabalho em equipe interprofissional, a efetivação das PC e a premissa seja que a interferência das Políticas Indutoras de Reorientação da Formação carrega o potencial de mudar paradigmas e coletivos, contudo, um aspecto importante está em destacarmos a desigualdade de acesso aos Programas que, ainda, ocorrem para uma minoria de discentes da graduação devido a prática dos Programas não estarem imbricadas nos projetos pedagógicos dos cursos das IES, mas sim atrelados à incentivos do governo federal. Este fato nos impede de poder apontar a interprofissionalidade enquanto tendências da Formação em Saúde, limitandoas apenas ao campo das experiências exitosas.

Situação também expressa nos depoimentos dos ex-discentes: 
A experiência do PET foi a grande responsável pelo meu aprendizado durante a graduação. Viver o cotidiano de trabalho e poder ser parte das trocas sociais e cientificas foi fundamental para minha formação. Todos deveriam ter a chance de viver isso" (Ex11).

As dificuldades nesse programa sempre irão acontecer, mas acredito que nunca serão maiores que a potência, pois até as dificuldades são aprendizado e possiveis reflexões e estudo. Minha sugestão é que nunca se pare de pensar políticas para garantir que mais e mais estudantes possam ser petianos como eu fui (Ex9).

"Após terminar graduação e atuando enquanto Residente Multiprofissional percebo a potência e o privilégio que tive quando participei do PET/Pró-Saúde" (EX9).

Os ganhos para se abraçar o Pró-Saúde e PET-Saúde estão no fortalecimento da integração ensino-serviço-comunidade e no estímulo efetivo por reformas curriculares. A mudança da forma de pensar dos atores sociais é percebida e reflete no aumento do potencial resolutivo das equipes com base no diagnóstico situacional do território, no desenvolvimento de competências e na introdução do componente-pesquisa envolvendo discentes, docentes e equipes dos serviços. E todos esses aspectos fazem eco diretamente na qualificação do acesso e da assistência no SUS sem a elevação substancial dos custos do financiamento destes processos.

\section{Referências}

ANDRÉ, Marli Eliza Dalmazo Afonso de. Etnografia da prática escolar. 2a ed. São Paulo: Papirus Editora, 1998.

AUSUBEL, David. Aquisição e retenção de conhecimentos: Uma perspectiva cognitiva. Lisboa: Editora Plátano, 2003.

BARR, Hugh; GRAY, Richard; HELME, Morion; LOW, Helena; REEVES, Scott. Interprofessional Education Guideline. CAIPE. Fareham, England, 2016.

, Hugh. Interprofessional education today, yesterday and tomorrow: a review. London, UK: Higher education academy, health sciences and practice network, 2005. Disponível em: Acesso em: 30. 07. 2017.

,Hugh. The Genesis of a Global Movement. In: Interprofessional Education. CAIPE. Fareham, England, 2015.

BORDENAVE, Juan Díaz; PEREIRA, Adair Martins. Estratégias de Ensino Aprendizagem. 29. ed. Rio de Janeiro: Vozes, 2008.

BRASIL. Ministério da Saúde. Política Nacional de Educação Permanente em Saúde. Série B. Textos Básicos de Saúde. Série Pactos pela Saúde: Brasília: 2009.

. Ministério da Saúde. Política Nacional de Educação Permanente em Saúde: o que se tem produzido para o seu fortalecimento?. Brasília: Ministério da Saúde, 1. Ed. Ver, 2018. 
CIHC. CANADIAN INTERPROFESSIONAL HEALTH COLLABORATIVE. A National Interprofessional Competency Framework. College of Health Disciplines, University of British Columbia. Vancouver, Canadá, 2010.

CONEJERO; Juana Suárez et al. Competencias esenciales em salud pública: un marco regional para las Américas. Rev Panam Salud Publica, v 34, n. 1, 2013.

CRUZ, Ynaiara Kristhine Stopa da; POLETTO, Rodrigo de Souza; MACHADO, Thaynara Aparecida; ALVES; Dayanne da Silva. Educação ambiental crítica na formação de professores: uma revisão sistemática de literatura. ENCITEC - Ensino de Ciências e Tecnologia em Revista, vol.11, n.1., p. 50-64, jan./abr. 2021.

ERICKSON, Frederick. Métodos cualitativos de investigación sobre la enseñanza. In M. Wittrok. La investigación de la enseñanza, II. Barcelona: Paidós. pp. 195-301, 1989.

FABRIGAR, Leonardo; WEGENER, Duane. Attitude structure. In: BAUMEISTER, R.F.; FINKEL, E.J. Advanced Social Psychology: the state of the science. United States of America: Oxford, 2010. chapter 7, p. 177-203.

FEUERWERKER, Laura Camargo Macruz; SENA-CHOMPRÉ, Roseni Rosangela. Interdisciplinaridade, trabalho multiprofissional e em equipe. Sinônimos? Como se relacionam e o que têm a ver com a nossa vida? Olho Mágico (Londrina), Londrina, v. 5, n. 18, p. 5-6, 1999.

FREIRE, Paulo. Pedagogia do Oprimido. Rio de Janeiro: Paz e Terra, 2005.

IPEC. INTERPROFESSIONAL EDUCATION COLLABORATIVE EXPERT PANEL. Core Competencies for Interprofessional Collaborative Practice. In: Report of an Expert Panel. Washington, 2011.

JANSEN, Paula. Validação Estatística e Análise Quantitativa. Resultados do instrumento Likert aplicado na tese de doutorado intitulada "Formação em Saúde para o Trabalho em Equipe: A Educação Interprofissional no âmbito do Pró-Saúde e PET-Saúde". Constructo Consultoria Integrada; São Paulo, 2017.

LEMOS, Evelyse dos Santos. A aprendizagem significativa: estratégias facilitadoras e avaliação. Periódico do Mestrado em Educação da UCDB, Campo Grande, v. 21, n. 1, p. 53-66, jan/jun. 2006.

MAHLER, Cornelia et al. Internal consistency of the readiness for interprofessional learning scale in German health care students and professionals. BMC Medical Education, v.14, 2014. Disponível em: http://www.biomedcentral.com/1472-6920/14/145. Acesso em 08 de abr. 2015.

MASINI, Elcie F. Salzano; MOREIRA, Marco Antonio. Aprendizagem significativa: condições para ocorrência e lacunas que levam a comprometimentos. São Paulo: Vetor, 2008.

MEDEIROS, Nara Maria Holanda de. Educação Permanente em Saúde: Gestão e Ensino na Concepção dos Trabalhadores. São Paulo: Fap-Unifesp, 2015.

Nara Maria Holanda de; MACHIN, Rosana. Educação Permanente como dispositivo de cogestão: a concepção dos profissionais de saúde da família do Conselho de Acompanhamento da Atenção Básica (CONACO). 2011. 169f. Mestrado Profissional em Ensino em Ciências da 
Saúde - Programa de Pós-Graduação em Ensino em Ciências da Saúde da Universidade Federal de São Paulo, UNIFESP, São Paulo, 2011.

Nara Maria Holanda de; Formação em saúde para o trabalho em equipe: a Educação Interprofissional no âmbito do Programa Nacional de Reorientação da Formação Profissional em Saúde (Pró-Saúde) e Programa de Educação pelo Trabalho para Saúde (PET-Saúde). 2019. $211 \mathrm{f}$. Doutorado em Ciências da Saúde - Programa de Pós-graduação Interdisciplinar em Ciências da Saúde da Universidade Federal de São Paulo, UNIFESP, Santos, 2019.

MENDRAS, Henri. O que é sociologia? São Paulo: Manole, 2004.

MOREIRA, Marco Antonio. Aprendizagem Significativa Crítica/Aprendizaje Significativo Crítico. Porto Alegre: Marco Antonio Moreira, 2005.

Marco Antonio; MASINI, Elcie. F. Salzano. Aprendizagem Significativa: a teoria de David Ausubel. São Paulo: Centauro, 2001.

Marco Antonio. Metodologias de Pesquisa em Ensino. São Paulo: Editora Livraria da Física, 2011.

Marco Antonio. A teoria de Aprendizagem Significativa segundo Ausubel In: MASINI, Elcie. F. Salzano (Org) Aprendizagem Significativa: condições para ocorrência e lacunas que levam a comprometimentos.1a ed. São Paulo: Vetor, 2008. p. 15-43.

MORIN, Edgar. Introdução ao Pensamento Complexo. Tradução Eliane Lisboa. 4. ed. Porto Alegre: Sulina, 2011.

OMS. ORGANIZAÇÃO MUNDIAL DA SAÚDE. Marco para Ação em Educação Interprofissional e Prática Colaborativa. Genebra, 2010. Disponível em: Acesso em: 31 jul. 2017.

PEDUZZI, Marina. O SUS é interprofissional. Interface - Comunicação, Saúde, Educação (Botucatu). São Paulo, v.20, no 56, 2016.

POSTMAN, Neil; WEINGARTNER, Charles. Contestação - Nova fórmula de ensino. 3 ed. Rio de Janeiro: Expressão e Cultura, 1969.

RITZ, Maria Rita de Cássia. Qualidade de Vida no Trabalho: Construindo, Medindo e Validando uma Pesquisa. Instituto de Matemática, Estatística e Computação Cientifica (IMECC), UNICAMP, Campinas; 2000.

SARGEANT, Joan. Theories to aid understanding and implementation of interprofessional education. J Cont Edu Health Prof, v.29, n.3, Canadá, 2009.

SCHIMIDT, Marty. Understanding and Using Statistics Basic Concepts. Massachusetts, USA: D.C. Health and Company. 1975.

VILELA, Elaine Morelato; MENDES, Iranilde José Messias. Interdisciplinaridade e Saúde: Estudo Bibliográfico. Rev. Latino-Am. Enfermagem, Ribeirão Preto, v. 11, n. 4, Aug. 2003.

XYRICHIS, Andreas; REAM, Emma. Teamwork: a concept analysis. Journal of advanced nursing. Wiley Online Library, 2008. Disponível em: https://doi.org/10.1111/j.1365- 2648.2007.04496.x. Acesso em: 06.03.2008. 